Research, Society and Development, v. 11, n. 1, e9911124382, 2022

(CC BY 4.0) | ISSN 2525-3409 | DOI: http://dx.doi.org/10.33448/rsd-v11i1.24382

\title{
Compatibilidade de inseticidas com o fungo entomopatogênico Metarhizium rileyi
}

\author{
(Ascomycota) \\ Compatibility of inseticides with the entomopathogenic fungus Metarhizium rileyi (Ascomycota) \\ Compatibilidad de insecticidas com el hongo entomopatógeno Metarhizium rileyi (Ascomycota)
}

Recebido: 06/12/2021 | Revisado: 13/12/2021 | Aceito: 19/12/2021 | Publicado: 02/01/2022

Luis Gustavo Amorim Pessoa

ORCID: http://orcid.org/0000-0003-4646-062X

Universidade Federal de Mato Grosso do Sul, Brasil

E-mail: luis.pessoa@ufms.br

Elisângela de Souza Loureiro

ORCID: http://orcid.org/0000-0002-9708-3775

Universidade Federal de Mato Grosso do Sul, Brasil

E-mail: elisangela.loureiro@ufms.br

Lidiane Arissa Yokota

ORCID: https://orcid.org/0000-0001-5478-9930

Universidade Federal de Mato Grosso do Sul, Brasil

E-mail: lidiane.yokota@ufms.br

Bruno Mateus Ribeiro Dias

ORCID: https://orcid.org/0000-0001-5315-8184

Universidade Federal de Mato Grosso do Sul, Brasil

E-mail: brunomateusagro@gmail.com

Tatiana Souza do Amaral

ORCID: htpps://orcid.org/0000-0001-5674-4998

Universidade Federal de Mato Grosso do Sul, Brasil

E-mail: taty.samaral@gmail.com

Marcelo Bruno Pessoa

ORCID: https://orcid.org/0000-0002-2601-8511

Universidade Federal de Mato Grosso do Sul, Brasil

E-mail: mbpessoa@gmail.com

Amanda Ramos Araújo

ORCID: https://orcid.org/0000-0002-4545-9128

Universidade Federal de Mato Grosso do Sul, Brasil

E-mail: amandars267@gmail.com

Gabriela Souza Oliveira

ORCID: https://orcid.org/0000-0001-5928-1351 Universidade Federal de Mato Grosso do Sul, Brasil

E-mail: gabrielasouzams14@gmail.com

Lauani Gabrieli Fidelis de Almeida

ORCID https://orcid.org/0000-0001-9066-7535

Universidade Federal de Mato Grosso do Sul, Brasil

E-mail: lauanifidelis2019@gmail.com

\begin{abstract}
Resumo
Atualmente existem no mercado inúmeras substâncias químicas empregadas no controle de insetos-praga. Entre eles, os inseticidas são um grupo numeroso e destacado. Este trabalho teve como objetivo avaliar a compatibilidade "in vitro" entre os inseticidas Permetrina (Pounce®) e Acefato (Orthene®) e os isolados UFMS 06 e UFMS 07 do fungo Metarhizium rileyi. Para tanto, o crescimento micelial, produção e germinação dos esporos foram avaliados. Ao solidificar o meio de cultura contendo os inseticidas, foram inoculados o fungo $M$. rileyi e incubados por 7 dias, em câmara climatizada a $25 \pm 1^{\circ} \mathrm{C}$, umidade relativa $70 \pm 10 \%$ e fotoperíodo de 12 horas. Para germinação dos esporos o período de incubação foi de 20 horas, nas mesmas condições citados anteriormente $\mathrm{O}$ índice biológico foi utilizada para o estabelecimento da classificação toxicológica dos inseticidas sobre o fungo. Os resultados mostraram que os produtos testados foram compatíveis com o fungo, podendo ser, portanto, utilizados no manejo integrado de pragas agrícolas por não afetarem o desenvolvimento do agente de controle biológico $M$. rileyi.
\end{abstract}

Palavras-chave: Controle biológico; Controle associado; Fungo entomopatogênico; Agricultura sustentável. 


\begin{abstract}
Currently, there are numerous chemical substances on the market used to control insect pests. Among them, insecticides are a numerous and prominent group. The objective of this work was to evaluate "in vitro" compatibility between insecticides Permethrin (Pounce $®)$ ) and Acephate (Orthene $®)$ ) and strains UFMS 06 and UFMS 07 of the fungus Metarhizium rileyi. Mycelial growth, spore production, and spore germination were evaluated. After solidifying the culture medium containing the insecticides, the fungus $M$. rileyi was inoculated and incubated for 7 days in an acclimatized chamber at $25 \pm 1{ }^{\circ} \mathrm{C}, 70 \pm 10 \%$ relative humidity, and 12 -hour photoperiod. For spores' germination, the incubation period was 20 hours, under the same conditions mentioned above. Biological index was used to establish the toxicological classification of insecticides on the fungus. The results showed that the products tested were classified as compatible, being compatible with the entomopathogenic fungus. Therefore, the products tested are recommended for use in the integrated management of agricultural pests as they do not affect the development of the biological control agent $M$. rileyi.
\end{abstract}

Keywords: Biological control; Associated control; Entomopathogenic fungus; Sustainable agriculture.

\title{
Resumen
}

Actualmente, existen en el mercado numerosas sustancias químicas utilizadas para el control de plagas de insectos, entre las cuales los insecticidas son un grupo numeroso y destacado. El objetivo de este trabajo fue evaluar "in vitro" la compatibilidad entre los insecticidas Permetrina (Pounce®) y Acefato (Orthene®), para aislar UFMS 06 y UFMS 07 del hongo Metarhizium rileyi. Se evaluó el crecimiento micelial, la producción de esporas y la germinación de las esporas. Luego de solidificar el medio de cultivo que contenía los insecticidas, se inoculó el hongo $M$. rileyi y se incubó durante 7 días en cámara climatizada a $25 \pm 1{ }^{\circ} \mathrm{C}, 70 \pm 10 \%$ de humedad relativa y fotoperiodo de 12 horas. Para la germinación de las esporas, el período de incubación fue de 20 horas, en las mismas condiciones mencionadas anteriormente. Se utilizó el índice biológico para establecer la clasificación toxicológica de los insecticidas sobre el hongo. Los resultados mostraron que los productos probados fueron clasificados como compatibles, siendo compatibles con el hongo entomopatógeno. Por lo tanto, los productos probados se recomiendan para su uso en el manejo integrado de plagas agrícolas ya que no afectan el desarrollo del agente de control biológico $M$. rileyi.

Palabras clave: Control biológico; Control asociado; Hongo entomopatógeno; Agricultura sostenible.

\section{Introdução}

Atualmente existem no mercado inúmeras substâncias químicas empregadas no controle de pragas e doenças. Entre estes, os inseticidas são um grupo numeroso e destacado, o que requer a utilização de produtos que sejam mais seletivos, minimizando a interferência no equilíbrio entre pragas e inimigos naturais. O controle biológico utilizando fungos entomopatogênicos é uma técnica importante para diminuir o uso de produtos fitossanitários químicos, mantendo assim essas pragas em níveis populacionais aceitáveis (Conte et al., 2019). A conservação e a utilização de agentes de controle biológico dentro dos cultivos agrários é uma estratégia muito utilizada no manejo integrado de pragas, havendo assim a necessidade de se conhecer a ação dos produtos fitossanitários de origem química sobre os bioinseticidas, inimigo natural dos insetos-praga, e a partir daí determinar sua seletividade/compatibilidade (Batista Filho et al., 2003).

Os fungos entomopatogênicos são importantes agentes de controle de insetos-praga. O desenvolvimento da doença inicia-se quando os conídios germinam, dependendo das condições de temperatura e umidade do ambiente, e penetram no tegumento do inseto (Alves, 1998). Metarhizium (=Nomuraea) rileyi (Ascomycota: Clavicipitaceae) (Kepler et al., 2014) destaca-se no controle de lepidópteros-praga com aproximadamente 30 espécies registradas como suscetíveis (Ignoffo et al., 1975), sendo relatado na literatura sua ocorrência natural, principalmente sobre lagartas da família Noctuidae (V. H. D. Costa et al., 2015; Sujii et al., 2002). Nos últimos anos foi constatado epizootia em lagartas da espécie S. cosmioides no Estado de Mato Grosso do Sul (Dias et al., 2019; Lima et al., 2015).

Crescimento vegetativo, viabilidade e a conidiogênese dos fungos patogênicos pode ser afetada por alguns produtos fitossanitários químicos, inclusive com a possibilidade de alteração da composição genética, influenciando na sua capacidade de infecção (Alves \& Lopes, 2008). A inibição ou redução do crescimento vegetativo de bioinseticidas também pode estar associada ao aumento na dose de produtos fitossanitários (Pinto et al., 2012), reforçando que a alteração da dose 
Research, Society and Development, v. 11, n. 1, e9911124382, 2022

(CC BY 4.0) | ISSN 2525-3409 | DOI: http://dx.doi.org/10.33448/rsd-v11i1.24382

recomendada dos produtos químicos no preparo das misturas de caldas contendo bioinseticidas aumenta as chances de

incompatibilidade (Santos et al., 2021).

Segundo Sosa-Gómez (2005), o efeito inibitório ou não dos inseticidas depende da espécie de fungo, uma vez que esse autor verificou que o produto Karate ${ }^{\circledR}$ em sua dose máxima se mostrou tóxico para o fungo $M$. rileyi e compatível com Metarhizium anisopliae (Metsch.) Sorok. A forma de exposição do fungo aos inseticidas também pode proporcionar respostas diferentes. Simulando diferentes formas de contato entre fungos entomopatogênicos e inseticidas, Carniel (2014) verificou que a exposição do fungo antes ou depois da aplicação de inseticidas promoveu resultados melhores na compatibilidade em comparação a mistura do fungo com o inseticida. Dessa forma, verifica-se que estratégias promovendo espaço temporal entre aplicações de inseticidas e fungos entomopatogênicos podem favorecer estes microrganismos.

A simples utilização de inseticidas compatíveis com biosinseticidas representa uma estratégia prática e econômica que contribui para a proteção dos entomopatógenos e equilíbrio do ambiente. À vista disso, este trabalho teve por objetivo testar a compatibilidade de alguns inseticidas utilizados e registrados na cultura da soja com o fungo entomopatogênico Metarhizium rileyi.

\section{Metodologia}

O presente estudo seguiu a metodologia de pesquisa laboratorial quantitativa proposta por Pereira et al. (2018). O efeito "in vitro" de inseticidas sobre os isolados UFMS 06 e UFMS 07 do fungo M. rileyi foi analisado por meio da avaliação do crescimento micelial, produção de esporos e germinação, na presença e ausência dos produtos fitossanitários, adicionando estes últimos ao meio de cultura BDA fundido, nas doses médias registradas pelos fabricantes (Tabela 1).

Tabela 1. Inseticidas utilizados no experimento de compatibilidade aos isolados UFMS 06 e UFMS 07 de Metarhizium rileyi, com seus respectivos ingredientes ativos e dose e volume de aplicação.

\begin{tabular}{cccc}
\hline Nome Comercial & Ingrediente Ativo & Dose & Volume de Aplicação \\
\hline Orthene $^{\circledR}$ & Acefato & $0,35 \mathrm{Kg}^{\circledR} \mathrm{ha}^{-1}$ & $200{\mathrm{~L} . \mathrm{ha}^{-1}}^{-}$ \\
Pounce $^{\circledR}$ & Permetrina & $52,5 \mathrm{~mL} \cdot \mathrm{ha}^{-1}$ & $200 \mathrm{~L}^{-\mathrm{ha}^{-1}}$ \\
\hline
\end{tabular}

Fonte: Agrofit (2021).

\subsection{Avaliação do crescimento micelial e concentração de esporos de Metarhizium rileyi}

Para avaliar o crescimento micelial e a produção de esporos do fungo, os inseticidas foram adicionados, em dosagem equivalente, ao meio de cultura (Tabela 1). Para evitar a degradação do inseticida, este foi adicionado ao meio de cultura apresentando 40 a $50^{\circ} \mathrm{C}$ de temperatura (Tanzini et al., 2002).

Posteriormente, a mistura foi vertida em placas de Petri de $9 \mathrm{~cm}$ de diâmetro, com o meio ainda não solidificado. Após a solidificação, a massa micelial do fungo foi inoculada em três pontos equidistantes na placa com auxílio de uma alça de platina. As placas foram então identificadas e lacradas com filme PVC e colocadas em câmara de incubação a $25 \pm 1{ }^{\circ} \mathrm{C}$, fotoperíodo de $12 \mathrm{~h}$ e umidade relativa de $70 \pm 10 \%$, por um período de sete dias.

Passados sete dias de incubação, o crescimento micelial do fungo foi avaliado por meio da medição de colônias previamente marcadas na parte externa da placa, A mediação foi feita em sentidos ortogonais para determinar o diâmetro médio, em centímetros, das colônias. Para análise da produção de esporos, as colônias marcadas foram recortadas das placas 
Research, Society and Development, v. 11, n. 1, e9911124382, 2022

(CC BY 4.0) | ISSN 2525-3409 | DOI: http://dx.doi.org/10.33448/rsd-v11i1.24382

com auxílio de um bisturi cirúrgico esterilizado e transferidas para tubos de ensaio esterilizado, contendo uma mistura de 10 $\mathrm{mL}$ de água destilada esterilizada e espalhante adesivo (Tween $80^{\circledR}$ ). Este foi agitado em agitador automático para a separar os agregados. Logo depois, os esporos foram contados em microscópio óptico com auxílio da câmara de Neubauer.

O delineamento do experimento foi inteiramente casualizado, composto por 3 tratamentos (testemunha, inseticida Orthene ${ }^{\circledR}$ e inseticida Pounce®) para cada isolado de $M$. rileyi (UFMS 06 e UFMS 07). Cada bioensaio consistiu de três placas contendo três colônias por placa. Do total de nove colônias por bioensaio, seis foram aleatoriamente selecionadas, que resultou em seis repetições por tratamento (Pessoa et al., 2020). Os dados de crescimento micelial e da produção de conídios foram transformados para $(\mathrm{x}+1)^{0,5}$ e submetidos ao teste de agrupamento de médias de Scott-Knott a $5 \%$ de probabilidade.

\subsection{Porcentagem de germinação}

A viabilidade dos esporos foi avaliada mediante a porcentagem de conídios germinados, utilizando uma suspensão de $1,0 \times 10^{6}$ con. $\mathrm{mL}^{-1}$ (Silva et al., 2005). Inicialmente, foi realizada raspagem da massa micelial e esporos de colônias de cada isolado de M. rileyi, anteriormente cultivado em BDA, sem inseticidas. Após a raspagem, os esporos foram colocados em tubos de ensaio contendo $10 \mathrm{~mL}$ água esterilizada + espalhante adesivo (Tween $80^{\circledR}$ ) e agitados para homogeneizar a suspensão. Um mL dessa suspensão original foi transferida para outro tubo de ensaio contendo 9 mL de água esterilizada + espalhante adesivo (Tween $80^{\circledR}$ ) juntamente com os inseticidas nas dosagens equivalentes ao volume de água utilizado. Esta suspensão foi mantida em repouso por duas horas. Seguidamente um mL desta suspensão foi plaqueada em placa de Petri e espalhada com alça de Drigalsky no meio de cultura. Logo depois, as placas foram vedadas com filme PVC e colocadas em uma câmara de incubação a $25 \pm 1{ }^{\circ} \mathrm{C}$, fotoperíodo de 12 horas e umidade relativa de $70 \pm 10 \%$ por 24 horas.

Após o período de incubação, foi realizada a avaliação da germinação. As placas foram divididas em quatro quadrantes, nos quais foram contados aleatoriamente 100 conídios, entre germinados e não germinados, por quadrante. Em seguida foi estimada a média por placa, por meio da qual calculou-se a porcentagem de germinação. Para avaliar a germinação, foi utilizado o padrão do laboratório do controle biológico do Instituto Biológico de Campinas, no qual: germinação alta 80$100 \%$, germinação média/alta 60-79 \%, germinação média 50-59 \%, germinação média/baixa 30-49 \% e germinação Baixa 0$29 \%$ (Zappelini et al., 2005).

O delineamento experimental foi inteiramente casualizado, consistiu em três tratamentos (testemunha, inseticida Orthene ${ }^{\circledR}$ e inseticida Pounce ${ }^{\circledR}$ ) para cada isolado de $M$. rileyi (UFMS 06 e UFMS 07), composto por três repetições por tratamento. Os dados de germinação foram transformados para arcsen (x/100) ${ }^{0,5}$ e submetido a análise da variância, sendo as médias posteriormente comparadas pelo teste de Scott-Knott a 5\% de probabilidade.

\subsection{Compatibilidade de inseticidas com o fungo entomopatogênico Metarhizium rileyi}

A classificação dos produtos em classes de seletividade/compatibilidade foi feita utilizando o fator de compatibilidade (IB = índice biológico), proposto por Rossi-Zalaf et al. (2008), conforme o efeito observado nos parâmetros avaliados anteriormente. Os valores obtidos para o IB foram comparados com os seguintes limites pré-estabelecidos: 0 a 41 = tóxico; 42 a 66 = moderadamente tóxico; > 66 = compatível.

\section{Resultados e Discussão}

\subsection{Crescimento micelial e produção de conídios}

O inseticida Orthene ${ }^{\circledR}$ não influenciou o crescimento micelial e a produção de esporos do isolado UFMS 06, bem como o crescimento micelial do isolado UFMS 07 de $M$. rileyi (Tabelas 1 e 2). No entanto, influenciou negativamente a 
produção de esporos do isolado UFMS 07 de M. rileyi (Tabela 2). Por outro lado, o inseticida Pounce® influenciou negativamente o crescimento micelial e produção de esporos do isolado UFMS 06 de $M$. rileyi, assim como a produção de esporos do isolado UFMS 07 (Tabelas 1 e 2).

Costa et al. (2018) relataram que o inseticida/acaricida Orthene ${ }^{\circledR} 75$ BR proporcionou menor crescimento do isolado UFMS 03 de M. rileyi, no entanto os inseticidas Mospilan ${ }^{\circledR}$, Imidacloprid ${ }^{\circledR}$ Nortox e Evidence ${ }^{\circledR} 700$ WG proporcionaram crescimento vegetativo semelhante a testemunha. Além do crescimento vegetativo, o inseticida Orthene®, bem como o Mospilan®, diminuíram significativamente a produção de conídios, enquanto que os inseticidas Imidacloprid®Nortox e Evidence ${ }^{\circledR} 700 W G$ não afetaram a conidiogênese. Tem sido demonstrado em vários estudos que o acefato, ingrediente ativo do inseticida Orthene®, diminui significativamente a produção de conídios de fungos do gênero Metarhizium (M. A. D. Costa et al., 2018; Duarte et al., 2016; Loureiro et al., 2002). Estes resultados mostram que a ação dos inseticidas sobre o crescimento micelial e a produção de esporos, varia em função da natureza do ingrediente ativo. De acordo com Morris (1975), produtos com a presença de emulsificantes e outros aditivos contribuem com a incompatibilidade de inseticidas aos entomopatógenos, representando um fator importante a ser controlado na elaboração de novas formulações comerciais de produtos.

As respostas ao efeito de inseticidas variam entre os microrganismos, e como pudemos observar no presente estudo, também podem variar de acordo com o isolado do fungo, no qual o inseticida Orthene® afetou a produção de esporos do isolado UFMS 07, mas não afetou o isolado UFMS 06 (Tabelas 2 e 3). É possível que o microorganismo, como resistência fisiológica, metabolize princípios tóxicos do ingrediente ativo como nutrientes secundários, permitindo deste modo um crescimento micelial e produção de esporos nos meios de culturas inoculados (Moino-Jr. \& Alves, 1998). Outra hipótese é que o fungo aumente seu esforço reprodutivo na presença de um evento negativo, como a presença de uma substância tóxica, levando a um crescimento micelial e produção de esporos ampliada (Loureiro et al., 2002). De acordo com Roberts \& Campbell (1977) a suscetibilidade de fungos entomopatogênicos a produtos químicos varia bastante entre os inseticidas e os diferentes isolados, o que pode explicar as diferenças de resultados obtidos por diferentes autores, assim como neste estudo.

Tabela 2. Crescimento micelial, produção de esporos, germinação do isolado UFMS06 do fungo entomopatogênicos Metarhizium rileyi na ausência (Testemunha) e presença dos inseticidas Orthene ${ }^{\circledR}$ e Pounce ${ }^{\circledR}$, e os valores obtidos para o índice biológico determinando a classificação de seletividade/compatibilidade dos inseticidas analisados.

\begin{tabular}{cccccc}
\hline Tratamento & $\begin{array}{c}\text { Crescimento } \\
\text { micelial }(\mathbf{c m})^{* *}\end{array}$ & $\begin{array}{c}\text { Produção de esporos } \\
\left(\times \mathbf{1 0}^{\mathbf{8}} \mathbf{c o n} \cdot \mathbf{m L}^{\mathbf{- 1}}\right)^{* *}\end{array}$ & $\begin{array}{c}\text { Germinação }(\%) \\
\text { Índice }\end{array}$ & $\begin{array}{c}\text { Classificação } \\
\text { biológico }\end{array}$ \\
\hline Testemunha & $2,92 \mathrm{a}$ & $2,88 \mathrm{a}$ & $84,49 \mathrm{a}$ & - & - \\
Orthene $^{\circledR}$ & $2,91 \mathrm{a}$ & $2,85 \mathrm{a}$ & $78,08 \mathrm{~b}$ & 98,67 & COMPATÍVEL \\
Pounce $^{\circledR}$ & $2,27 \mathrm{~b}$ & $1,83 \mathrm{~b}$ & $89,16 \mathrm{a}$ & 74,39 & COMPATÍVEL \\
\hline $\mathrm{CV}(\%)$ & 4,90 & 13,87 & 6,60 & - & -
\end{tabular}

Valores seguidos pela mesma letra, na coluna, não diferem significativamente entre si, pelo teste de Scott-Knott ao nível de 5\% de probabilidade. *Para análise dados transformados em $\operatorname{arcsen}(\mathrm{x} / 100)^{0,5} * *$ Para análise dados transformados em $(\mathrm{x}+1)^{0,5}$ Fonte: Autores (2021). 
Tabela 3. Crescimento micelial, produção de esporos, germinação do isolado UFMS07 do fungo entomopatogênicos Metarhizium rileyi na ausência (Testemunha) e presença dos inseticidas Orthene ${ }^{\circledR}$ e Pounce ${ }^{\circledR}$, e os valores obtidos para o índice biológico determinando a classificação de seletividade/compatibilidade dos inseticidas analisados.

\begin{tabular}{|c|c|c|c|c|c|}
\hline Tratamento & $\begin{array}{c}\text { Crescimento } \\
\text { micelial }(\mathbf{c m}) * *\end{array}$ & $\begin{array}{c}\text { Produção de } \\
\text { esporos } \\
\left(\times 10^{8} \text { con.mL } L^{-1}\right)^{* *}\end{array}$ & Germinação $(\%)^{*}$ & $\begin{array}{c}\text { Índice } \\
\text { biológico }\end{array}$ & Classificação \\
\hline Testemunha & $3,05 \mathrm{a}$ & $4,21 \mathrm{a}$ & $81,52 \mathrm{a}$ & - & - \\
\hline Orthene ${ }^{\circledR}$ & $2,83 \mathrm{a}$ & $3,06 \mathrm{~b}$ & $40,08 \mathrm{~b}$ & 79,75 & COMPATÍVEL \\
\hline Pounce $^{\circledR}$ & $2,75 \mathrm{a}$ & $2,05 \mathrm{c}$ & $89,83 \mathrm{a}$ & 74,37 & COMPATÍVEL \\
\hline $\mathrm{CV}(\%)$ & 9,50 & 19,04 & 9,98 & - & - \\
\hline
\end{tabular}

Valores seguidos pela mesma letra, na coluna, não diferem significativamente entre si, pelo teste de Scott-Knott ao nível de 5\% de probabilidade. *Para análise dados transformados em arsen $(\mathrm{x} / 100)^{0,5} * *$ Para análise dados transformados em $(\mathrm{x}+1)^{0,5}$ Fonte: Autores (2021).

\subsection{Germinação}

Quanto à germinação dos isolados UFMS 06 e UFMS 07, o inseticida Pounce ${ }^{\circledR}$ não diferiu da testemunha, ao contrário do inseticida Orthene ${ }^{\circledR}$, que diminuiu a germinação dos fungos (Tabelas 2 e 3). Apesar do inseticida Pounce ${ }^{\circledR}$ ter diminuído o crescimento do isolado UFMS 06, e a produção de conídios de ambos os isolados, a germinação dos conídios não foi afetada, o que indica que não houve redução na viabilidade dos conídios. Entretanto o inseticida Orthene ${ }^{\circledR}$, que não afetou o crescimento micelial de ambos isolados, e nem a produção de conídios do isolado UFMS 06, diminuiu a germinação de ambos os isolados, o que indica que ele afeta a viabilidade dos conídios de $M$. rileyi, demonstrando também a importância de analisar todas as fases importantes do ciclo de vida dos fungos estudados.

\subsection{Compatibilidade de inseticidas ao fungo entomopatogênico Metarhizium rileyi}

O fator de compatibilidade (IB) ficou entre 98,67 e 74,37 para os dois inseticidas estudados, sendo, portanto, classificados como compatíveis por não inibirem o desenvolvimento do fungo entomopatogênico. Podemos considerar que estes inseticidas também sejam compatíveis com o fungo em campo, visto que estudos "in vitro" expõem o microrganismo ao máximo à ação do químico estudado, diferente do campo onde diversos fatores podem servir para alterar as condições de exposição, atestando a inocuidade do produto em campo caso a mesma seja encontrada no experimento em laboratório. Outro fator a ser considerado é que mesmo uma alta toxicidade de um produto "in vitro" não significa uma alta toxicidade em campo, pelo mesmo motivo da interferência de fatores na exposição do microorganismo ao princípio ativo. Porém, esta toxicidade deve ser interpretada como uma possibilidade de danos ao microorganismo em campo (Alves, 1998; Moino-Jr. \& Alves, 1998).

\section{Conclusão}

Os inseticidas testados foram compatíveis com os isolados UFMS 06 e UFMS 07 de M. rileyi, podendo ser, portanto, utilizados no manejo integrado de pragas agrícolas por não afetarem o desenvolvimento do agente de controle biológico $M$. rileyi. Entretanto, estudos realizando avaliações em campo são necessários para melhor elucidar o potencial de compatibilidade ao fungo M. rileyi, uma vez que, como já mencionado, diversos fatores em campo não presentes nos experimentos "in vitro", 
Research, Society and Development, v. 11, n. 1, e9911124382, 2022

(CC BY 4.0) | ISSN 2525-3409 | DOI: http://dx.doi.org/10.33448/rsd-v11i1.24382

bem como a natureza do ingrediente ativo do inseticida, podem afetar as condições de exposição e a resposta dos fungos aos inseticidas.

\section{Agradecimentos}

A Coordenação de Aperfeiçoamento de Pessoal do Nível Superior (CAPES) pela concessão da bolsa de estudo. Este estudo foi financiado em parte pela Coordenação de Aperfeiçoamento de Pessoal de Nível Superior - Brasil (CAPES), Código de Financiamento 001, e pelo Conselho Nacional de Desenvolvimento Científico e Tecnológico (CNPq), Código de Financiamento 001; Universidade Federal de Mato Grosso do Sul (UFMS), pelos recursos para publicar este manuscrito; aos membros do grupo de pesquisa LAMIP.

\section{Referências}

Alves, S. B. (1998). Controle microbiano de insetos (2.ed.). FEALQ.

Alves, S. B., \& Lopes, R. B. (2008). Controle microbiano de pragas na América Latina: Avanços e desafios. FEALQ.

Batista Filho, A., Ramiro, Z. A., Almeida, J. E. M., Leite, L. G., Cintra, E. R. R., \& Lamas, C. (2003). Manejo integrado de pragas em soja: Impacto de inseticidas sobre inimigos naturais. Arquivos do Instituto Biológico, 70, n.1, 61-67.

Conte, O., Silveira, J. M., Possamai, E. J., \& Harger, N. (2019). Resultados do monitoramento integrado da colheita da soja na safra $2018 / 2019$ no Paraná (Circular Técnica $\mathrm{N}^{\circ}$ 157; Embrapa Soja. Circular técnica, 157, p. 26p.). Londrina: Embrapa Soja. https://ainfo.cnptia.embrapa.br/digital/bitstream/item/212108/1/CIRCULAR-TECNICA-157-.pdf

Costa, M. A. D., Loureiro, E. D. S., Pessoa, L. G. A., \& Dias, P. M. (2018). Compatibilidade de inseticidas utilizados na cultura do eucalipto com Metarhizium rileyi (FARLOW) (=Nomuraea rileyi). Revista de Agricultura Neotropical, 5(3), 44-48. https://doi.org/10.32404/rean.v5i3.2149

Costa, V. H. D., Soares, M. A., Rodríguez, F. A. D., Zanuncio, J. C., da Silva, I. M., \& Valicente, F. H. (2015). Nomuraea rileyi (Hypocreales: Clavicipitaceae) in Helicoverpa armigera (Lepidoptera: Noctuidae) larvae in Brazil. Florida Entomologist, 98(2), 796-798.

Dias, P. M., Loureiro, E. D. S., Pessoa, L. G. A., Mateus, M. P. de B., Tosta, R. A. de S., Oliveira Neto, F. M. de, \& Devoz, G. L. R. (2019). Epizootia de Metarhizium rileyi em Helicoverpa armigera (Lepidoptera: Noctuidae) em cultivo de soja. In Anais: Resumos da $71^{a}$ Reunião Anual da SBPC, UFMS, Campo Grande, Brasil/Sociedade Brasileira para o Progresso da Ciência (p. 4pp). SBPC. http://livro.sbpcnet.org.br/71ra/index.htm

Duarte, R. T., Gonçalves, K. C., Espinosa, D. J. L., Moreira, L. F., De Bortoli, S. A., Humber, R. A., \& Polanczyk, R. A. (2016). Potential of entomopathogenic fungi as biological control agents of diamondback moth (Lepidoptera: Plutellidae) and compatibility with chemical insecticides. Journal of Economic Entomology, 109(2), 594-601.

Ignoffo, C. M., Futtler, B., Marston, N. L., Hostetter, D. L., \& Dickerson, W. A. (1975). Seasonal incidence of the entomopathogenic fungus Spicaria rileyi associated with noctuid pests of soybeans. Journal of Invertebrate Pathology, 25(1), 135-137.

Kepler, R. M., Humber, R. A., Bischoff, J. F., \& Rehner, S. A. (2014). Clarification of generic and species boundaries for Metarhizium and related fungi through multigene phylogenetics. Mycologia, 106(4), 811-829.

Lima, A. de, Loureiro, E. S. L., Muchalak, F., Taira, T. L., Ferreira, F. N., \& Nocchi, M. J. (2015). Ocorrência de Nomuraea rileyi (Farlow) Samson na Spodoptera cosmioides (Walk.) 1858 (Lepidoptera: Noctuidae) em Chapadão do Sul-MS. Tecnologia \& Ciência Agropecuária, 9(2), 57-59.

Loureiro, E. D. S., Moino Jr., A., Arnosti, A., \& Souza, G. C. D. (2002). Efeito de produtos fitossanitários químicos utilizados em alface e crisântemo sobre fungos entomopatogênicos. Neotropical Entomology, 31(2), 263-269. https://doi.org/10.1590/S1519-566X2002000200014

Moino-Jr., A., \& Alves, S. B. (1998). Efeito de imidacloprid e fipronil sobre Beauveria bassiana (Bals.) Vuill. E Metarhizium anisopliae (Metsch.) Sorok. E no comportamento de limpeza de Heterotermes tenuis (Hagen). Anais da Sociedade Entomológica do Brasil, 27(4), 611-619. https://doi.org/10.1590/S030180591998000400014

Morris, O. N. (1975). Effect of some chemical insecticides on the germination and replication of commercial Bacillus thuringiensis. Journal of Invertebrate Pathology, 26(2), 199-204.

Pereira, A. S., Shitsuka, D. M., Parreira, F. J., \& Shitsuka, R. (2018). Metodologia da Pesquisa Científica [recurso eletrônico] (1. ed.). UFSM, NTE. https://repositorio.ufsm.br/bitstream/handle/1/15824/\%20Lic_Computacao_MetodologiaPesquisa-Cientifica.pdf?sequence=1

Pessoa, L. G. A., Souza, T. M. N., \& Loureiro, E. de S. (2020). Compatibilidade de inseticidas utilizados no manejo de pragas em eucalipto com Beauveria bassiana (Cordycipitaceae). Research, Society and Development, 9(8). https://doi.org/10.33448/rsd-v9i8.5148

Pinto, L. M. N., Dörr, N. C., Ribeiro, A. P. A., Salles, S. M. de, Oliveira, J. V. de, Menezes, V. G., \& Fiuza, L. M. (2012). Bacillus thuringiensis monogenic strains: Screening and interactions with insecticides used against rice pests. Brazilian Journal of Microbiology, 43(2), 618-626. https://doi.org/10.1590/S151783822012000200025

Roberts, D. W., \& Campbell, A. S. (1977). Stability of entomopathogenic fungi. Miscellaneous Publications of the Entomological Society of America, 10, 1976. 
Research, Society and Development, v. 11, n. 1, e9911124382, 2022

(CC BY 4.0) | ISSN 2525-3409 | DOI: http://dx.doi.org/10.33448/rsd-v11i1.24382

Rossi-Zalaf, L. S., Alves, S. B., Lopes, R. B., Silveira Neto, S., \& Tanzini, M. R. (2008). Interação de microrganismos com outros agentes de controle de pragas e doenças. In Controle microbiano de pragas na América Latina: Avanços e desafios (2.nd., p. 270-302). FEALQ.

Santos, C. A. M., Nascimento, J., Gonçalves, K. C., Smaniotto, G., Freitas Zechin, L., Costa Ferreira, M., \& Polanczyk, R. A. (2021). Compatibility of Bt biopesticides and adjuvants for Spodoptera frugiperda control. Scientific Reports, 11(1), 5271. https://doi.org/10.1038/s41598-021-84871-w

Silva, R. Z. da, Neves, P. M. de O. J., \& Santoro, P. H. (2005). Técnicas e parâmetros utilizados nos estudos de compatibilidade entre fungos entomopatogênicos e produtos fitossanitários. Semina: Ciências Agrárias, 26(3), 305. https://doi.org/10.5433/1679-0359.2005v26n3p305

Sosa-Gómez, D. R. (2005). Seletividade de agroquímicos para fungos entomopatogênicos [Folhetos]. Londrina: Embrapa Soja. http://ainfo.cnptia.embrapa.br/digital/bitstream/CNPSO-2009-09/28931/1/seletiv_fung.pdf

Sujii, E. R., Tigano, M. S., \& Sosa-Gomes, D. (2002). Simulação do impacto do fungo Nomuraea rileyi em populações da lagarta da soja, Anticarsia gemmatalis. Pesquisa Agropecuária Brasileira, 37(11), 1551-1558. https://doi.org/10.1590/S0100-204X2002001100005

Tanzini, M. R., Alves, S. B., \& Setten, A. (2002). Toxicidade de produtos fitossanitários utilizados no controle de Leptopharsa heveae para fungos entomopatogênicos. Arquivos do Instituto Biológico, 69(4), 65-69.

Zappelini, L. O., Almeida, J. E. M., \& Gassen, M. H. (2005). Compatibilidade de fungos entomopatogênicos com emulsificantes para óleo vegetal e pó molhável. Arquivos do Instituto Biológico, 72, 1-63. 